
costa central del Ecuador

María Quiñónez-Macías, Kervin Chunga, José Torres, Williams Méndez. 


\title{
Evaluación de riesgo costero y registros climáticos MIS 1 a MIS 3 en la costa central del Ecuador
}

\section{Coastal hazard assessment and climate change record since MIS-3, central coast of Ecuador}

María Quiñónez-Macías ${ }^{(* 1)}$, Kervin Chunga ${ }^{(2-3)}$, José Torres ${ }^{(2)}$, Williams Méndez ${ }^{(3)}$

(1) Secretaría de Gestión de Riesgos, Dirección de Análisis de Riesgos, Ecuador.

(2) Universidad Estatal Península de Santa Elena, UPSE, Facultad de Ciencias de la Ingeniería, Ecuador.

(3) Universidad Técnica de Manabí, Departamento de Construcciones Civiles, Portoviejo, Manabí, Ecuador * maria.quinonez@gestionderiesgos.gob.ec

\begin{abstract}
Resumen
Los análisis estratigráficos, biológicos y geoquímicos han proporcionado suficiente información para reconstruir la evolución paleográfica y climática de la costa del cantón Jaramijó. Uno de los resultados más notables, es la identificación y delineación de un paleo-acantilado costero de edad $\left({ }^{14} \mathrm{C}\right) 43.245 \pm 460$ B.P. (perteneciente al MIS-3). Este MIS-3 es asociado a una época de glaciación, pero los datos obtenidos e interpretados en este estudio nos indican que la costa central del Ecuador registra un nivel interestadial (años cálidos en una etapa glacial). Dos acantilados más han sido cartografiados desde análisis de orthofotos, pero estos son de menor edad. La reciente transgresión holocénica ha modificado la costa central del Ecuador e incrementando el nivel de riesgo costero climático por las variaciones del nivel de mar. Paleo-línea de costas han sido evidenciados en los veriles -5.5m y-7.6m, a 440 y 650 m de distancias de la línea de costa actual. Para el sitio de Jaramijó, la tasa de retroceso de los acantilados está en el orden de 1.1 a 2.4 m/año. Estas tasas de erosión pueden incrementarse si se consideran modelos matemáticos que estiman un escenario en el 2100 de incrementos del nivel de mar en +1 y $+1.4 m$.
\end{abstract}

Palabras Clave: Retroceso de acantilados, riesgos climáticos, variación nivel de mar, Jaramijó.

\begin{abstract}
Stratigraphic, biological and geochemical analyzes have provided sufficient information to reconstruct the paleographic and climatic evolution of the coast of the Jaramijo canton. One of the most remarkable results is the identification and delineation of a paleo sea-cliff of age ${ }^{14} \mathrm{C} 43,245 \pm 460$ B.P. (belonging to the MIS-3). This MIS-3 is associated with a period of glaciation, but the data obtained and interpreted in this study indicate that the central coast of Ecuador has an interstadial phase (warm years in a glacial stage). Two more paleo-coastal cliffs have been mapped from orthofotos analysis, but these are younger. The Holocene transgression has modified the central coast of Ecuador and increased the level of coastal climate hazard by sea-level rise. Paleo-coastlines have been evidenced in the depth contours of $-5.5 \mathrm{~m}$ and $-7.6 \mathrm{~m}$, at 440 and $650 \mathrm{~m}$ distances from the up-to-date coastline. For the Jaramijo site, the rate of cliff-erosion and wave-cut platforms is in the order of 1.1 to $2.4 \mathrm{~m} / \mathrm{yr}$. These cliff-erosion rates can be increased if we consider mathematical models with an estimated sea-level rise scenario to be in 2100 of about +1 to $+1.4 m$.
\end{abstract}

Keywords: Cliff-retreat, coastal climate hazards, sea-level rise, Jaramijó. 


\section{Introducción}

El cambio climático comprende bien distinguidos procesos bioquímicos y mecánicos desarrollados en y encima de la corteza oceánica y continental. El incremento y descenso del nivel de mar, referido en este estudio para el Cuaternario, es el efecto más evidente de las variaciones climáticas y modelamiento del margen costero (Rohling et al., 2008; Revell et al., 2011). Todos estos efectos de cambios ocurridos en el pasado son registrados en los sedimentos y en los modelamientos (cambios de forma) del terreno, dependiendo de aportes sedimentarios o procesos de erosión, de las etapas glaciales e interglaciales (definido también como Marine Isotope Stage, MIS) (Emiliani, 1958; Shackleton, 1967; Chappell \& Shackleton, 1986; Martinson et al., 1987), y de las intensas precipitaciones o del desgaste por acción marina, fluvial y eólica.

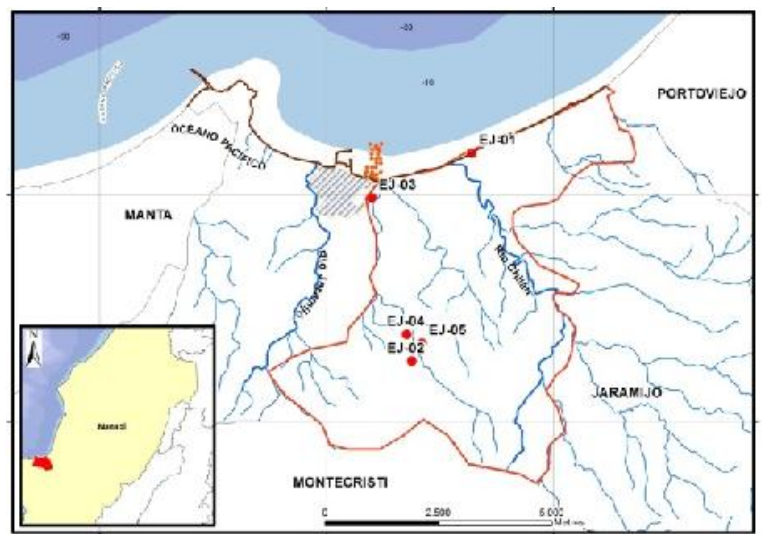

Figura 1. Ubicación del cantón Jaramijó y localización de las estaciones de muestreo al interno del continente y piso marino.

Las modificaciones en el terreno en su mayor parte son debidas a los aportes de sedimentos transportados desde el continente, como flujos de detritos por las intensas lluvias y por deslizamientos caóticos (ejemplo la bahía de Jaramijó en Manabí). Los aportes de sedimentos marinos hacia el continente son por avance del nivel del mar, conocido como transgresión holocénica.

Por otra parte, el nivel de mar puede incrementarse en un muy corto periodo durante los eventos ENSO en el orden de $60 \pm 20 \mathrm{~cm}$ (Cobb et al., 2003; Levitus et al., 2009), y son estos efectos locales los que aceleran la tasa de retroceso de los acantilados costeros, en particular donde los macizos rocosos son de suave consistencia como las arcillolitas o limolitas fracturadas (ie., soft bedrock), y en los sedimentos holocénicos. Para el Ecuador, esta información histórica inicia desde 1955, donde se han presentado eventos que prominentemente ocurren en 1957 a 1958 , 1969 a 1970, 1972 a 1973, 1982 a 1983 , y 1997 a 1998 (Levitus et al., 2009), y el evento más reciente de menor magnitud de 2015 a 2016. El evento El Niño de 1969 presenta un comportamiento inusual en el que a pesar de existir fuertes anomalías de velocidad de arrastre existe relativamente poco calentamiento (Mato and Toulkeridis, 2017).

Los periodos de recurrencias de las amenazas naturales asociados al cambio climático, han generado múltiples peligros geológicos a lo largo del tiempo (deMenocal, 2001), que ha modificado bruscamente el paisaje en la costa central del Ecuador (Mulas et al., 2015; Chunga et al., 2015). Es así, que en el terreno se han evidenciado los episodios históricos y prehistóricos que han desplazado culturas precolombinas costeras, ejemplo, Manteña, Jama-Coaque, Guangala, Chorrera, Machalilla y Valdivia (Zeidler and Pearsall, 1994; Usselman, 2006).

Tabla 1. Afloramientos de secuencias estratigráficas Cuaternarias y rasgos geomorfológicos.

\begin{tabular}{|l|l|l|l|}
\hline Estac. & X $(\mathrm{mE})$ & $\mathrm{Y}(\mathrm{mN})$ & Rasgo del terreno \\
\hline EJ-01 & 543208 & 9895924 & Acantilado de playa \\
\hline EJ-02 & 541879 & 9891333 & Paleo-acantilado \\
\hline EJ-03 & 550818 & 9888337 & Terraza aluvial \\
\hline EJ-04 & 545753 & 9890427 & Valle aluvial \\
\hline EJ-05 & 543767 & 9893072 & Colina alta \\
\hline
\end{tabular}

Para la reconstrucción paleoclimática y paleogeográfica, se ha seleccionado un sitio que cumple con todas las características sedimentarias anteriormente descrita, esta es la franja costera del cantón Jaramijó (provincia de Manabí), el cantón tiene un área de $96 \mathrm{~km}^{2}$, confinante con los otros cantones de Portoviejo, Montecristi y Manta (ver Figura 1). Secuencias de estratos cuaternarios bien preservadas han sido estudiadas en este estudio, y referidas como estaciones de muestreos EJ-01 a EJ-05 (Figura 1).

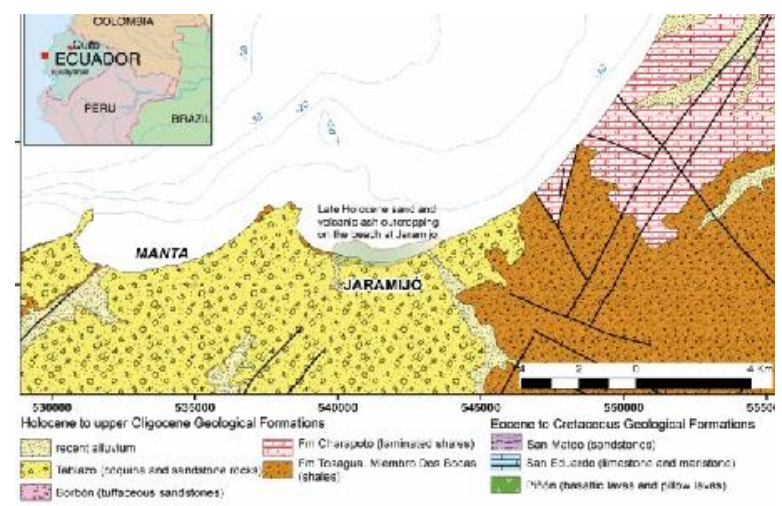

Figura 2. Extracto de mapa geológico de Manta a escala 1: 100.000, delineando los afloramientos de sedimentos y capas volcánicas de cenizas en el borde costero de Jaramijó (Bristow \& Hoffstetter, 1977). 
En este estudio se ha compilado información biológica (microorganismos y microfósiles, moluscos y bivalvos), geológica (tipo de relieve, sedimentos y estratos cuaternarios) y geoquímica (dataciones de ${ }^{14} \mathrm{C}$, isotopos estables de oxígenos y medidas de paleotemperaturas de los océanos desde análisis a restos de carbonos y conchas). Los datos que se utilizaron para analizar el cambio de la línea de costa son datos topográficos y batimétricos a escala de 1: 5.000. Estos datos analizados proporcionaron detalles de indicadores geomorfológicos y estratigráficos de los cambios climáticos desarrollados en esta zona central de la costa del Ecuador.

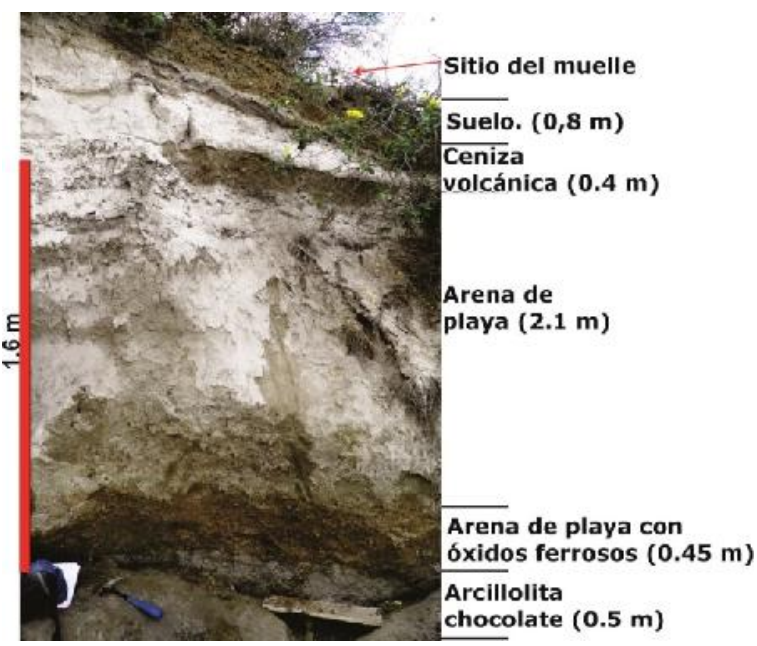

Figura 3. Afloramiento de sedimentos y rocas en la estación EJ-03, a un costado del muelle Ideal. Nótese el espesor de cada unidad litológica identificada en este estudio. La unidad inferior de arcillolita corresponde a roca, las superiores son deposiciones de sedimentos cuaternarios.

\section{Fase de investigación y método de estudio}

\subsection{Geomorfología costera y procesos erosivos}

El substrato rocoso es conformado por rocas de arcillolitas "chocolates" del Miembro Dos Bocas de la formación Tosagua (Figuras 2 y 3), encima, en algunos sectores de las plataformas de abrasión, afloran estratos discontinuos muy compactos de coquina correspondiente a la formación Tablazo (Figura 4), con espesores en el rango de 2 a 10 metros. Arcillas marinas del Pleistoceno superior con espesores que alcanzan hasta los 5 metros cubren las coquinas y arcillolitas. Posteriormente, sedimentos recientes que en margen costero tienen bien conservadas secuencias sedimentarías y volcánicas del Holoceno, en los acantilados costeros y también en cortes verticales por excavaciones de canteras al interno del cantón (ie., EJ02, EJ04m EJ05). Estos sedimentos son conformadas por arenas de playa con oxidación ferrosa y abundante moluscos (Figura 3), cubiertas por cenizas volcánicas de lahares secundarios y por deposición fall- out que alcanzan en algunas unidades litológicas los 0.6 $m$ de espesor (Mulas et al., 2015), por encima arenas grisáceas no cementada, que conforma la litología de muchos cordones litorales de la bahía de Jaramijó.

Toda esta secuencia estratigráfica, en particular los sedimentos Holocénicos indican un tipo de costa emergente con sedimentación progradacional, lo cual significa que esta franja costera se está levantando pocas fracciones de un milímetro cada año (ie., 0.5 mm/año; Pedoja et al., 2006) o por súbitos levantamientos tectónicos $(0.2$ a $0.3 \mathrm{~m})$, como recientemente documentados en el terremoto de Pedernales 2016, Mw 7.8 (Chunga et al., 2017). Las terminologías de transgresión y progradación dependen del avance o retiro del nivel de mar, cuando el nivel de mar se retira se denomina regresión y desde el continente se incrementa la tasa de sedimentación progradacional; mientras si el nivel de mar avanza hacia el continente hay aportes de sedimentos marinos por transgresión (Posamentier et al., 1992).
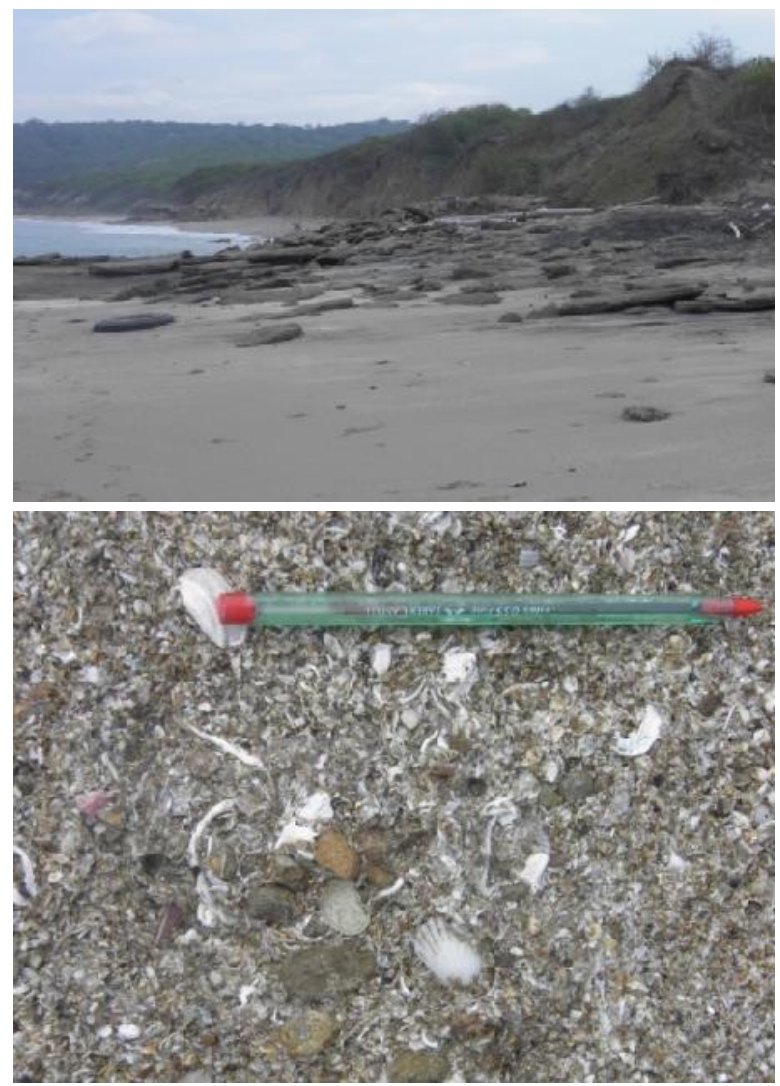

Figura 4. Afloramiento en la bahía de Jaramijó de estratos de coquina de la formación Tablazo, erosionados por la dinámica de las olas marinas. Coordenadas UTM $545.395 \mathrm{mE}, 9.897 .001 \mathrm{mN}$.

La costa de Jaramijó presenta escenarios de rápidos cambios atribuidos a los procesos marinos (dinámicas de olas y corrientes), estratigráficos (rocas altamente erosionables como la arenisca y limolita), tectónicos (fracturamiento en macizos rocosos y lineamientos estructurales que son los responsables del levantamiento o subsidencia) y morfológicos (terrazas 
marinas en etapa de erosión por procesos fluviales), que al combinarse con otros factores como los meteorológicos (precipitaciones, temperatura del aire, viento y humedad) ocasionan cambios muy bruscos en el relieve.
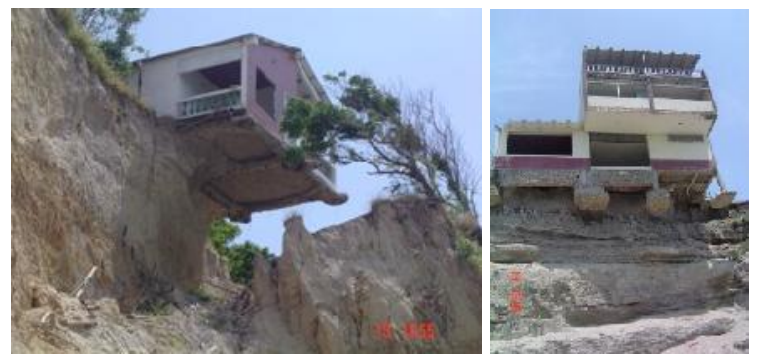

Figura 5. Afectación de viviendas por el retroceso del acantilado debido al incremento del nivel de mar (Soledispa, 2012).

Los acantilados que se evidencian en la costa se encuentran en proceso de rápida erosión marina formando las plataformas rocosas de abrasión que han quedado por el retroceso de los mismos, es decir las colinas fueron erosionadas y transformadas en planicies rocosas (Soledispa, 2012). Esto se debe a la acción del oleaje y al incremento del nivel del mar, que llegan de forma directa erosionando y desestabilizando los taludes, provocando deslizamientos de tierra en la zona de playa y estos a la vez transportados por las olas a otros sitos. Estos acantilados de baja a mediana altura y de playas arenosas con una pendiente de $5^{\circ}$ a $15^{\circ}$.

La bahía de Jaramijó presenta costas con colinas bajas, cortadas como acantilados costeros, con alturas entre 4 y $20 \mathrm{msnm}$, hacia el continente presenta colinas medias entre los 20 y $82 \mathrm{msnm}$. Adicionalmente, esta bahía también presenta colinas altas en los límites del cantón Montecristi, alcanzando alturas hasta $175 \mathrm{~m}$ (altitudes determinadas desde el análisis de DEM's, modelos digitales del terreno en una plataforma GIS). En Jaramijó, desde el pie del acantilado hasta las colinas altas, fueron encontradas niveles sedimentarios asociadas a una sedimentación de transgresión que posteriormente fueron levantadas por la tectónica activa de la región (Pedoja et al., 2006). Las terrazas marinas en el sitio presentan erosión dominadas por los factores climáticos como el exceso de altas precipitaciones que se presentaron en algún momento sobre la bahía de Jaramijó, que actualmente son evidentes en la superficie del terreno y en los cortes fluviales, los cuales fueron identificados también con las orthofotos a escala 1: 6.000 .

El paisaje geomorfológico tiende a ser combinado con la tectónica activa, ya que es habitual observar las terrazas marinas y sus estratos conformados de moluscos marinos, levantados con desniveles de altitudes en forma de escalera (Pedoja et al., 2006). Burbank \& Anderson (2001), mencionan que en escalas de tiempo del Holoceno estamos en una época postglacial y que las condiciones climáticas variaban poco respecto a las otras etapas interglaciales registradas en el pasado, estos ciclos climáticos pueden haber dejado huellas fuertes en el paisaje, como se evidencia en Jaramijó al observarse muy acentuadas incisiones fluviales y de rápida erosión en el relieve.
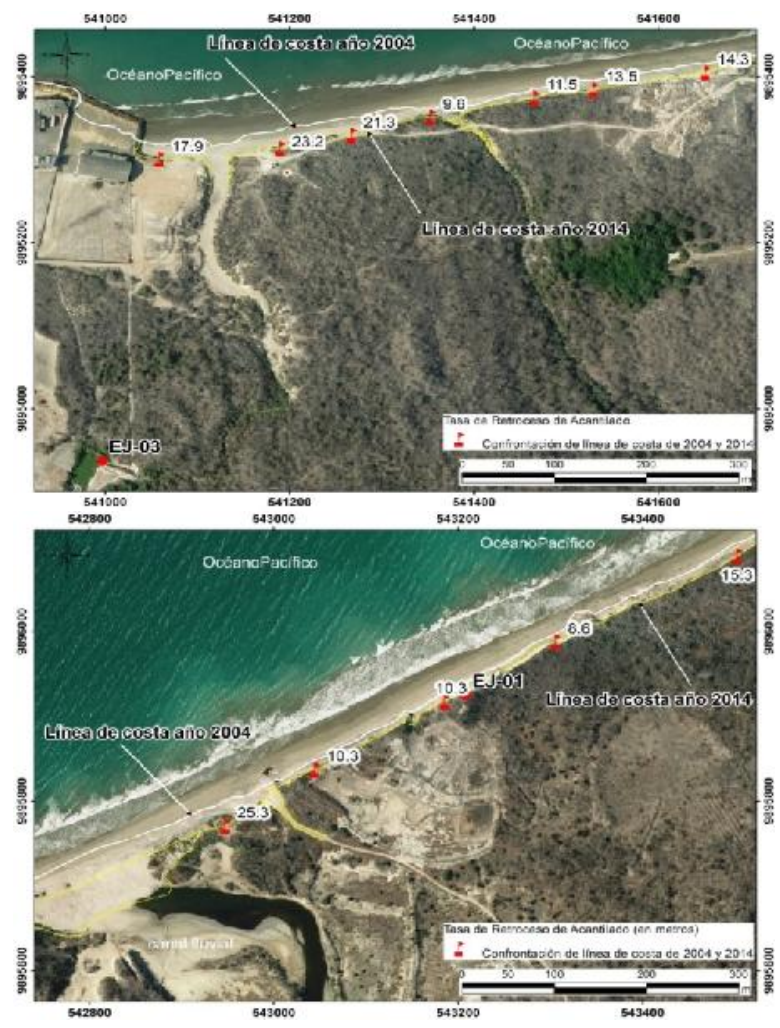

Figura 6. Tasa de retroceso (en metros) del acantilado en Jaramijó desde posición de las líneas de costas del 2004 y 2014.

\subsection{Retroceso de acantilados y transgresión Holocénica}

Durante la compilación de datos en el terreno, se pudo evidenciar múltiples deslizamientos a lo largo del margen costero, la dinámica de las olas marinas erosionan el acantilado costero que tiene una altura entre 10 a $18 \mathrm{~m}$, poco a poco colapsan la base del talud y de esta manera retroceden de su posición la línea de contacto océano- continente. En las instalaciones de la Base Naval de Jaramijó, BASJAR, hay evidencia de muelle destruido y muros de concretos arrancados desde sus cimentaciones por el socavamiento del talud debido al incremento del nivel de mar. Además, en esta franja costera (a excepción del muelle artesanal) no hay obras de ingeniería y construcciones de espigones para contrarrestar los efectos de dinámica de erosión por ondas marinas.

Este retroceso del acantilado (por transgresión holocénica) deja al descubierto nuevas evidencias estratigráficas bien conservadas de secuencias sedimentarias del Holoceno (Figura 5) y de niveles de 
cenizas volcánicas depositadas en dos sentidos (Figura 3 ), por lahares secundarios de cenizas y por depositación aérea (transporte de cenizas por acción del viento, fall-out).

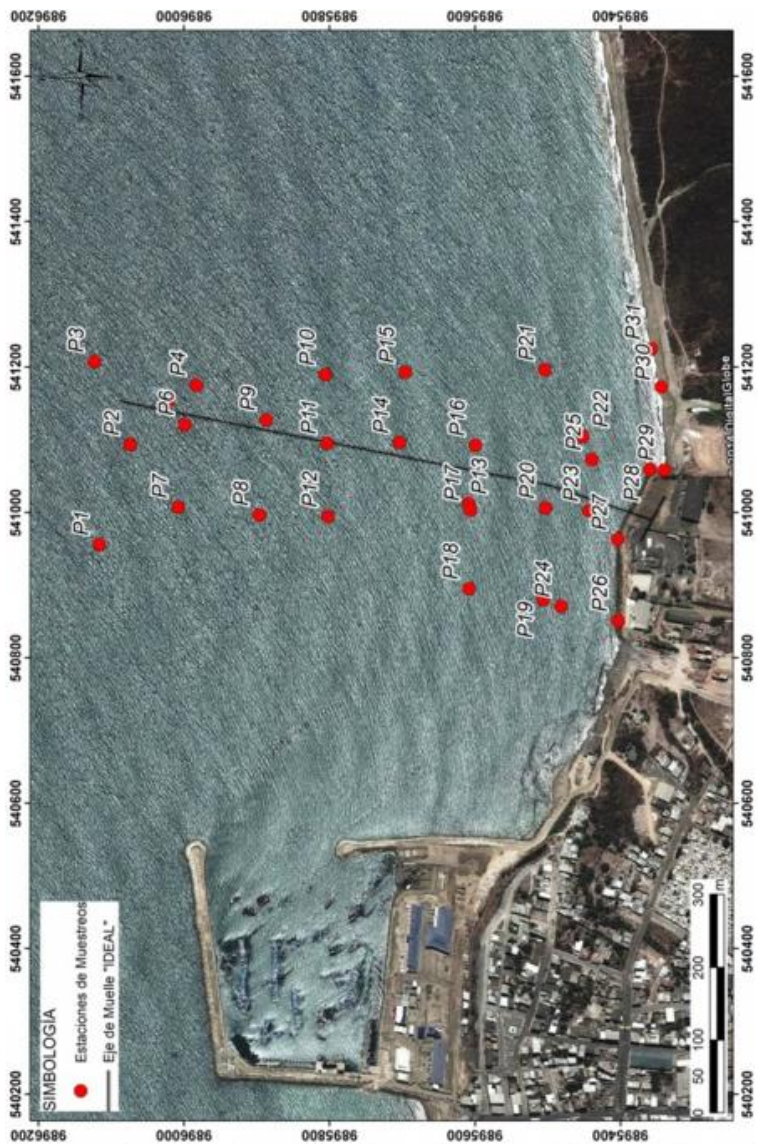

Figura 7. Localización de estaciones de muestras de sedimentos de fondos obtenidas con el instrumento "van veen", en el piso marino en la bahía de Jaramijó. Las muestras P26 a P31 corresponden a la zona de playa intertidal.

Datos topográficos realizados por el Inocar (desde el 2004 y 2014), a través del equipo GPS RTK Tramble permitieron obtener datos con precisión de altimetría en el terreno y proporcionados en literatura por Chunga et al. (2015), demuestran la evidencia propuesta en este estudio de retroceso del acantilado. Además, el análisis de fotos aéreas del año 1965 fue confrontada con las imágenes satelitales proporcionada por "Google earth" del año 2013, y es notorio este cambio de retroceso del acantilado. Todos estos datos topográficos de altimetría y de análisis de fotos aéreas han permitido establecer la rápida tasa de retroceso de este margen costero de 11 a 24 metros cada 10 años o dicho en otro sentido de 1.1 a $2.4 \mathrm{~m} /$ año, esto indica la rápida tasa de erosión del acantilado conformado litológicamente por rocas y sedimentos blandos y de fácil ripabilidad (Figura 6).

\subsection{Reconstrucción de paleocostas}

La obtención de 31 muestras de sedimentos en el piso marino de Jaramijó (Figura 7) (hasta el veril -8 metros debajo de nivel de mar) y en la zona intertidal, ha sido realizada empleando una lancha (Figura 8), con un GPS marca Magallan, de alta precisión. El GPS permitió ubicar las coordenadas UTM WGS 1984 datum $17 \mathrm{~S}$, a lo largo de la plataforma interna costera. La posición de coordenadas y toma de muestras tienen un mallado aproximado entre 50 y $100 \mathrm{~m}$. Todas estas muestras han sido enumeradas con las abreviaciones desde P1 hasta P31 (ver Figura 7).

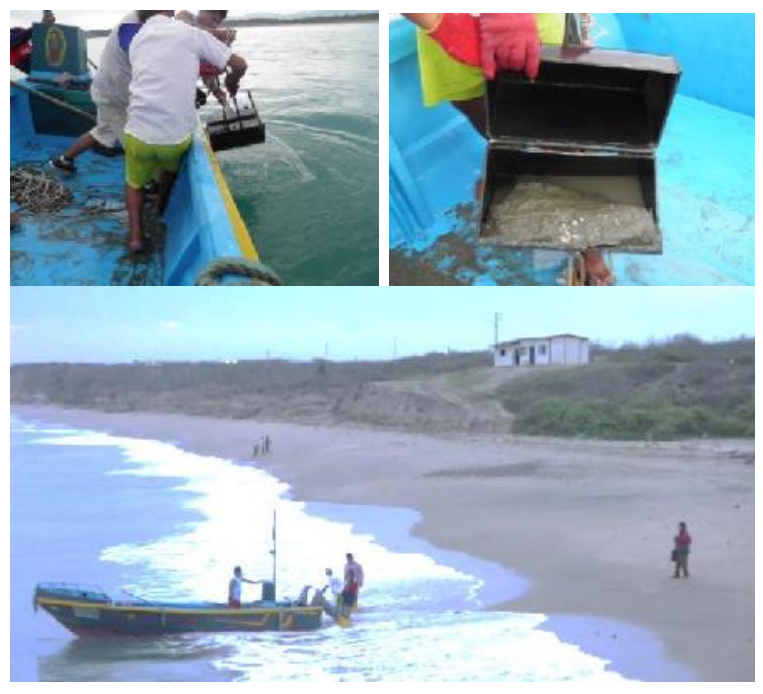

Figura 8. Embarcación de instrumento de muestreo de sedimento "van veen" para la extracción de muestras de sedimentos en el piso marino alcanzando el veril de -10 metros debajo del nivel de mar. Con la técnica peso y cuerda se determinó la profundidad de columna de agua.

Durante esta fase de campo también se determinó la columna de agua o profundidad (Z) desde la superficie al piso marino (ver Figura 8), utilizando el método de peso y conteo con cuerda seccionada en metros. Estos datos permiten generar un mapa de distribución de sedimentos de fondos (Figura 9) y de batimetría de detalle referido a la Figura 10. El trabajo en mar compila 25 muestras de sedimentos de fondo tipo limo, limo arenoso, arena limosa y arena media con abundantes moluscos, y 6 muestras de arena de playa en continente (ver Figura 7). Cabe recalcar, que durante la fase de extracción de muestras de sedimentos empleando el instrumento "van veen", no se obtuvo muestras en zonas rocosas, el poco material arenoso de grano grueso, indica que es roca de tipo coquina (perteneciente a la formación geológica Tablazo) que se encuentra en altos batimétricos del piso marino (ver Figura 9, mapa de distribución de sedimentos de fondos). Se deduce que estos estratos rocosos se encuentran cubiertos por una capa de poco espesor de sedimentos arenosos.

\section{Resultados}

\subsection{Mapa de distribución de sedimentos y paleo- línea de costa}

De acuerdo a los análisis macroscópicos de granulometría realizada a las 25 muestras obtenidas en 
el piso marino y a la posición de coordenadas UTM se ha procedido a generar un mapa de distribución de sedimentos. En la Figura 9, se puede observar un cambio sedimentológico a medida que nos adentramos al piso marino, en la línea de costa hay predominancia de arena de granulometría fina a media, la cual es representativa a lo largo de la franja de playa.

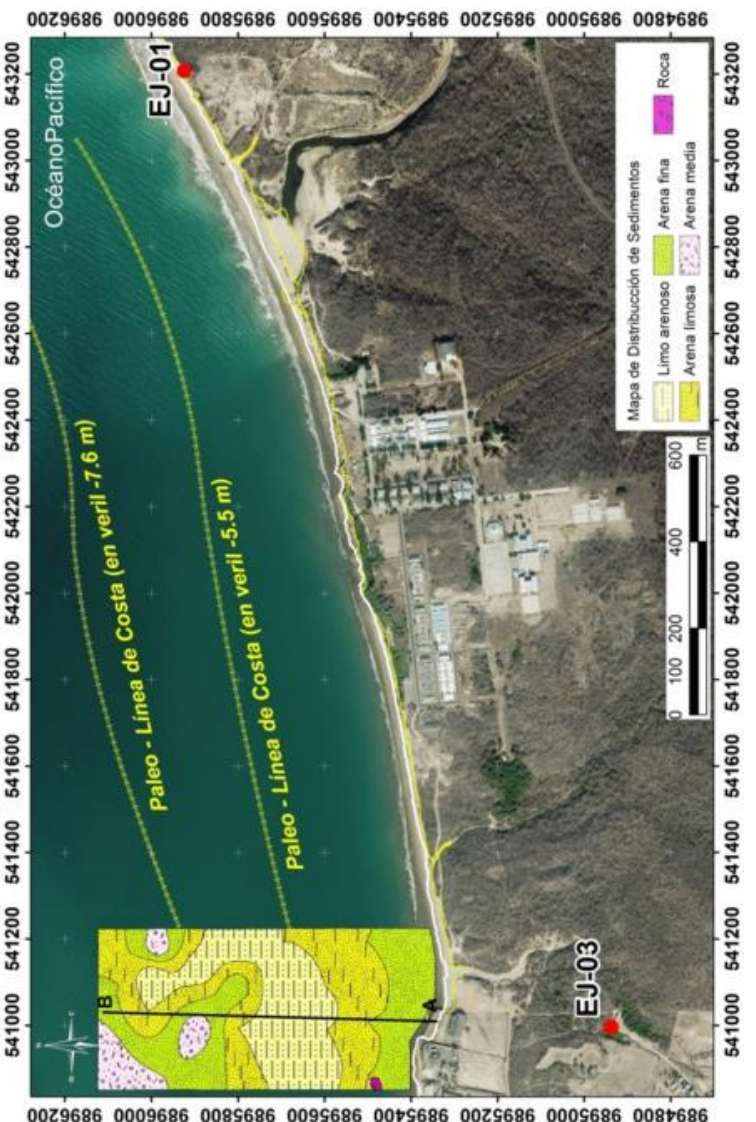

Figura 9. Mapa de distribución de sedimentos desde el veril 0 m.s.n.m. hasta los $-8 \mathrm{~m}$ y delineación de paleo-costas en los veriles -5.5 y $-7.6 \mathrm{~m}$.

En los veriles batimétricos (Figura 10) entre los -1.5 metros a $-3.5 \mathrm{~m}$ el sedimento es de arena limosa, reduciendo su granulometría a limo arenoso en los veriles $-3.5 \mathrm{~m}$ a $-5.5 \mathrm{~m}$. En el veril $-5.5 \mathrm{~m}$ a $-5.8 \mathrm{~m}$ una estrecha franja de arena limosa indica un incremento del grano de sedimento, desde los-5.8m a -6.2 hay predominancia de arena fina. Entre los veriles -6.2 a $7 \mathrm{~m}$ hay variaciones de sedimentos finos entre arena limosa y limo arenoso. Desde los $-7 \mathrm{~m}$ a $-8.2 \mathrm{~m}$ hay predominancia de sedimento arena limosa. Cabe recalcar que sedimentos de grano medio a grueso se encuentran al noroeste del sitio y un sector aislado a noreste entre los veriles $-7.5 \mathrm{~m}$ a $-8 \mathrm{~m}$. Altos rocosos en columna de agua somera (veril $-1 \mathrm{~m}$ a $1.5 \mathrm{~m}$ ) se encuentran al suroeste cercano a la línea de costa y al muelle artesanal de Jaramijó (marcado como roca en el mapa), representado en la Figura 9.

Con los datos de profundidad de columna de agua obtenida en la fase de campo, y aplicando el software Surfer10 y GIS ArcMap se ha procedido a interpolar valores de profundidad para la generación de un mapa batimétrico (Figura 10). El método de interpolación empleada en el software Surfer es la técnica "kriging". Para una mejor visualización del sitio de estudio se ha extraído una imagen satelital de google-earth que ha sido georreferenciado en una plataforma GIS con el programa ArcMap.

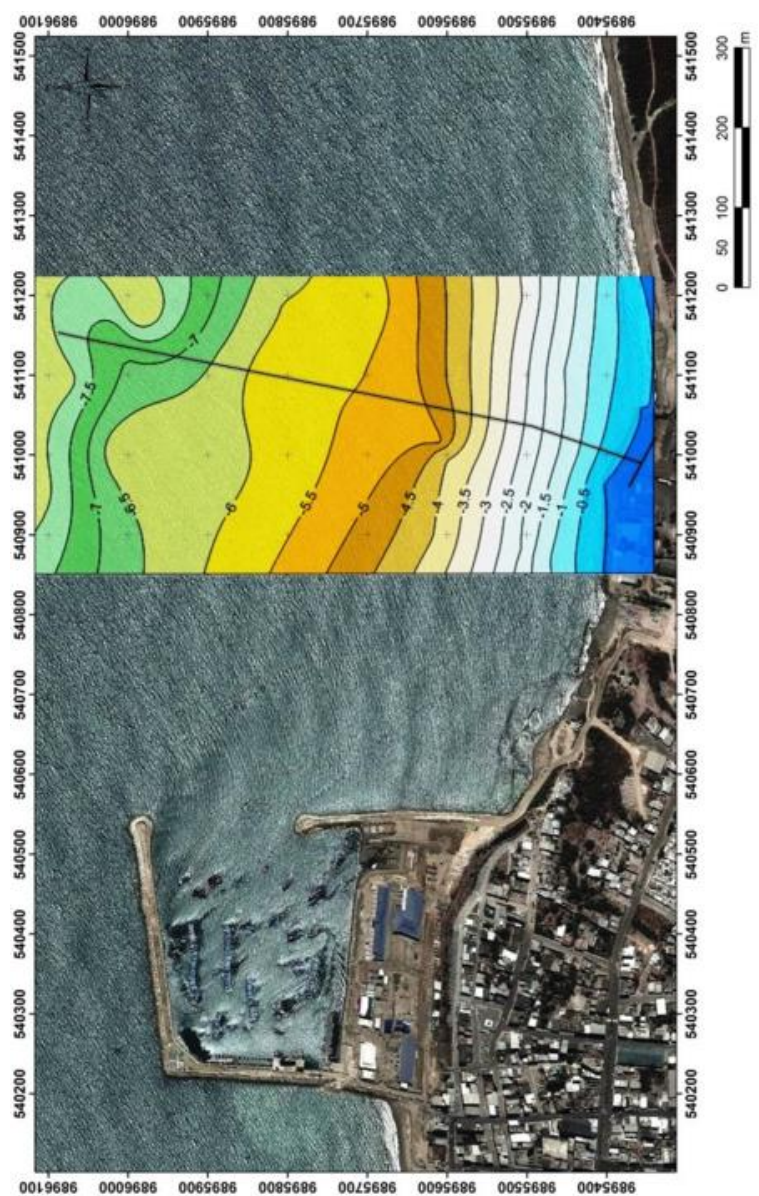

Figura 10. Mapa de batimetría en el sector del muelle Ideal y río Jaramijó. En el sector oeste se puede apreciar el muelle pesquero artesanal de Jaramijó. Los veriles de profundidad son expresados en metros.

En el perfil batimétrico A-B (Figura 12) se puede evidenciar que desde la línea actual de playa hasta los veriles $-5 \mathrm{~m}$ hay una franja estrecha geomorfológicamente atribuida a una zona de pendiente escarpada entre los $16^{\circ}$ a $35^{\circ}$ de inclinación. Desde los $-5 \mathrm{~m}$ hasta los $-6.5 \mathrm{~m}$ se presenta una terraza litoral de inclinación suave entre los 2 a $4^{\circ}$ posiblemente atribuida a una antigua línea de costa o paleo-costa (ver Figura 9). Desde los $-6.5 \mathrm{~m}$ a $-7 \mathrm{~m}$ el talud poco inclinado de pendiente moderadamente escarpada alcanza inclinaciones entre $\operatorname{los} 8^{\circ}$ a $16^{\circ}$. En el veril $-7 \mathrm{~m}$ se desarrolla una nueva terraza litoral estrecha de pendiente inclinada entre $\operatorname{los} 4^{\circ}$ a $8^{\circ}$, tal forma representa también una paleo costa. Desde los $7 \mathrm{~m}$ a -8.2 se evidencia nuevamente un talud moderadamente escarpado entre $\operatorname{los} 8^{\circ}$ a $16^{\circ}$ de inclinación (Figura 12). 


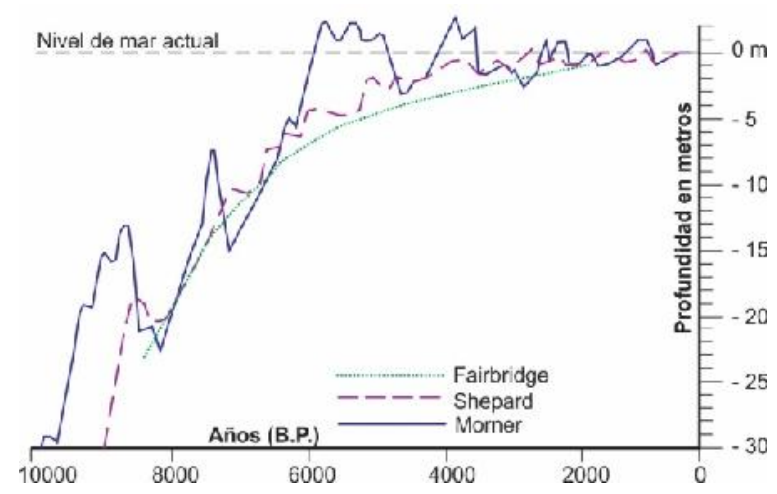

Figura 11. Transgresión holocénica y variación de nivel de mar en los últimos 10.000 años (Fairbridge, 1961; Shepard, 1963; Morner, 1982).

Interpretando este perfil batimétrico podemos indicar que: (a) las dos terrazas litorales son asociadas a paleo-líneas de costa y el talud inclinado es el incremento acelerado de elevación del nivel de mar, la distancia entre una de la otra es consecuencia de las variaciones registrada por la transgresión holocénica en el MIS-1 (Figura 11). Pero, algo significativo es evidenciado en el talud superior por el rápido incremento de nivel de mar próximo a la línea de costa actual (último avance de transgresión holocénica, Figura 12).

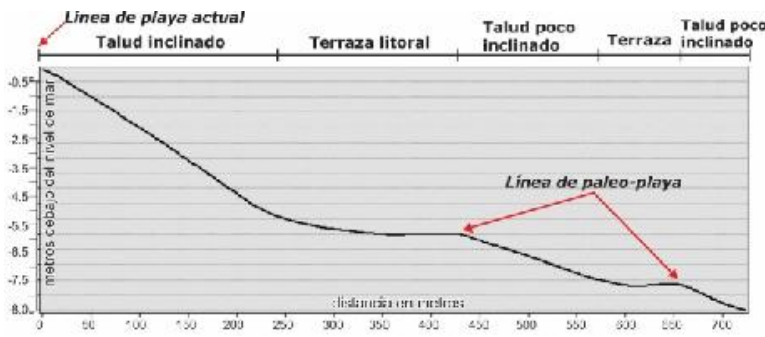

Figura 12. Perfil batimétrico del sitio Jaramijó (perfil A - B) iniciado en las coordenadas UTM $540.989 \mathrm{mE}$ $9.895 .358 \mathrm{mN}$ y finalizado en mar en las coordenadas $541.153 \mathrm{mE}-9.896 .088 \mathrm{mN}$, longitud total del perfil es de 760 metros (ver Figura 9). Nótese las paleo-líneas de costas en los veriles $-5.5 \mathrm{~m}$ y $-7.6 \mathrm{~m}$.

\subsection{Paleoambiente y reconstrucción paleoclimática}

Esta investigación se focaliza en reconstruir la evolución paleogeográfica y climática del sitio, cuyas depositaciones o aportes sedimentarios se presentaron durante el MIS 3 (rango de edades entre 59.000 a 24.000 B.P.) al MIS 1 (rango de 18.000 años atrás hasta el presente) (Martison et al., 1987).

El sitio de estudio Jaramijó tiene un alto registro paleoclimático donde se puede encontrar un paleoacantilado a $4 \mathrm{~km}$ de distancia de la línea de costa (Figura 13) levantado hasta la altitud 30 a 35 m.s.n.m., tales registros estratigráficos documenta un posible depósito interestadial (estación de muestreo EJ-02) de edad ${ }^{14} \mathrm{C} 43.245 \pm 460$ años BP.

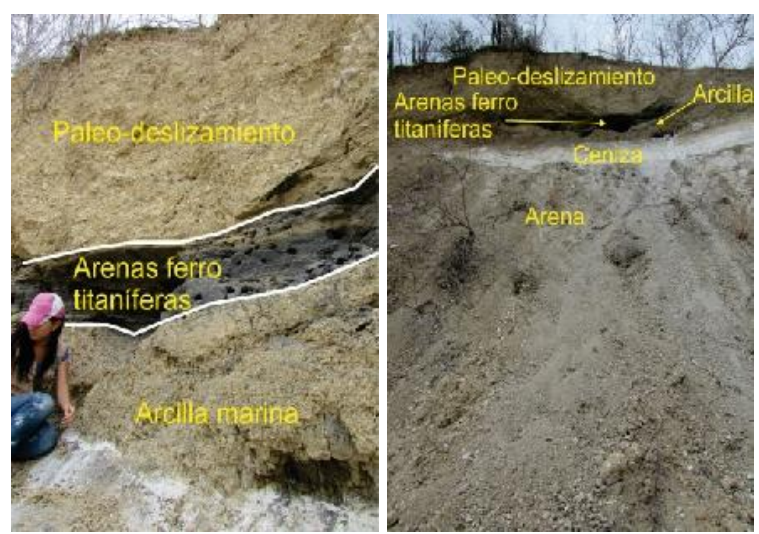

Figura 13.Unidades litológicas en estación EJ-02 depositadas en una zona de canal y paleo-acantilado, en la base arcilla marina.

Para delinear la paleo-costa de edad estimada $43.245 \pm 460$ años B.P. se empleó el uso de orthofotos y modelo digital del terreno en plataforma GIS, en la Figura 15 se pueden observar relieves de colinas medias con alturas entre 20 a 45 m.s.n.m. (paleoacantilados). Este paisaje fue rápidamente modificado por subsiguientes aportes de flujos de detritos o deslizamientos como los observados en la columna estratigráfica de la estación EJ-02 (Figura 13). Para comprender mejor la evolución paleogeográfica, se elaboró un perfil topográfico indicando las terrazas o levantamientos tectónicos de nuestro estudio, más aquella información proporcionada por Pedoja et al. (2006).

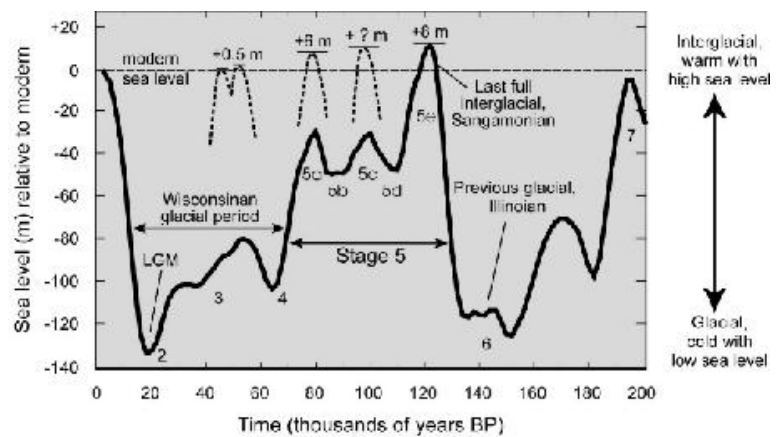

Figura 14. Delineación de los MIS (Marine Isotope Stages) durante los últimos 200.000 años y su relación de paleocostas y variaciones del nivel de mar (Martison et al., 1987).

Desde el corte de acantilado de la estación EJ-03 y de los localizados 4 kilómetros al interior del continente, de las estaciones EJ-02, EJ-04 y EJ-05 (ubicación en Figura 1), se evidenció un nivel arenoso con abundante restos de icnofacies (estructuras sedimentarias producto de la actividad orgánica). Los icnofacies son estructuras que reflejan la actividad de organismos como perforaciones, senderos, pistas, 
madrigueras y pellets, se utilizan como pistas para identificar algunas condiciones de depósito y además aportan información útil para la interpretación paleoambiental. El desarrollo de estas comunidades bentónicas es controlado por varios factores ecológicos como disponibilidad de oxígeno, la salinidad, temperatura, luminosidad, la consistencia del sustrato, la turbulencia y energía del agua de fondo, la velocidad del depósito $\mathrm{y}$, por los eventos que los alteran (tormentas, corrientes de turbidez, lluvia de ceniza) (Shackleton, 1978). Los icnofacies se preservan mejor en areniscas y arenas, tanto el tamaño de grano como las condiciones de depósito contribuyen a su preservación.

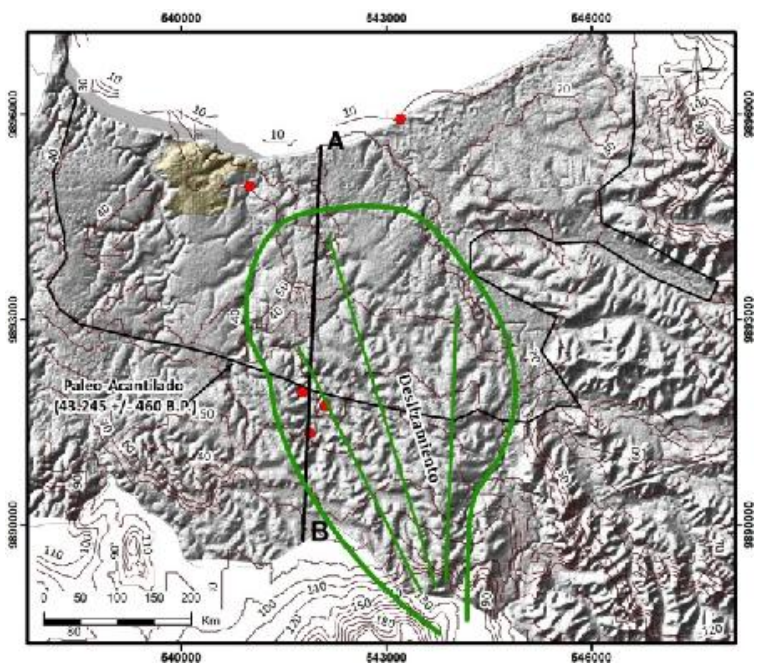

Figura 15. Localización de perfil topográfico A - B para la reconstrucción paleogeográfica. Paleoacantilado de edad 43.245 años \pm 460 B.P., marcado con línea negra con tendencia NW-SE.

En el perfil A-B de la Figura 15, este nivel arenoso de icnofacies puede representar la zona sublitoral acontecida probablemente durante el periodo de interglaciación del MIS 5e, cuando el nivel de mar alcanzaba los +8 metros por encima del nivel actual del mar (Figura 14), hace 120.000 años atrás. La tasa de levantamiento tectónico estimado para Jaramijó en este estudio, desde datos de altimetría confrontando con las edades de las terrazas y paleoacantilados costeros, es de $0.5 \mathrm{~mm} / a$ ño a $0.98 \mathrm{~mm} / a n ̃ o$ (Quiñónez-Macías, 2016). Pedoja et al. (2006), estima una tasa de $0.31 \mathrm{~mm} /$ año a $0.39 \mathrm{~mm} / \mathrm{año}$, considerando terrazas marinas de edades entre 120.000 años (MIS 5e) y 220.000 años (MIS 7) y 330.000 años (MIS 9).

Los niveles sedimentarios analizados comprenden depósitos acontecidos en el MIS 3 a MIS 1. El nivel de estrato arenoso EJ-2E tiene una datación de Cal. BC 41.295 a 40.140 años (Cal. BP 43.245 a 42.090 años). En la Figura 17 hacemos referencia a la edad 43.245 años \pm 460 B.P., donde la línea de costa de aquel tiempo se encontraba a 4 kilómetros de distancia de la costa actual.
Para una mayor compilación de datos para la reconstrucción paleogeográfica y dependiendo del sitio de estudio, se debe considerar el escenario geológico, el sitio de Jaramijó tiene aportes sedimentarios por las transgresiones marinas del periodo interglacial y aportes progradacionales significativos durante los periodos proglaciales (después de la terminación de la glaciación). Este último aporte puede estar asociado a los vastos deslizamientos coluviales, de flujos de detritos o de lahares secundarios de cenizas provenientes desde las colinas medias y altas del sector sur y sureste del cantón Jaramijó (Figura 15).

Secuencias estratigráficas fueron erosionadas en el terreno sin embargo las restante unidades litológicas aflorantes en las estaciones de muestreos han permitido reconstruir terrazas más recientes desde el Pleistoceno superior al Holoceno, como la T1 a una altitud de 20 m.s.n.m, terraza T2 a una altitud entre los 30 m.s.n.m. y la T3 a una altitud entre los 43 a 57 m.s.n.m (Chunga et al., 2016; Quiñónez-Macías, 2016). Es importante indicar que esta última terraza T3 es considerada por Pedoja el at. (2006), y que esta investigación ha delineado dos nuevas terrazas las cuales en su mayor parte se encuentra cubierta por deslizamiento de lahares secundarios de cenizas volcánicas (Mulas et al., 2015)
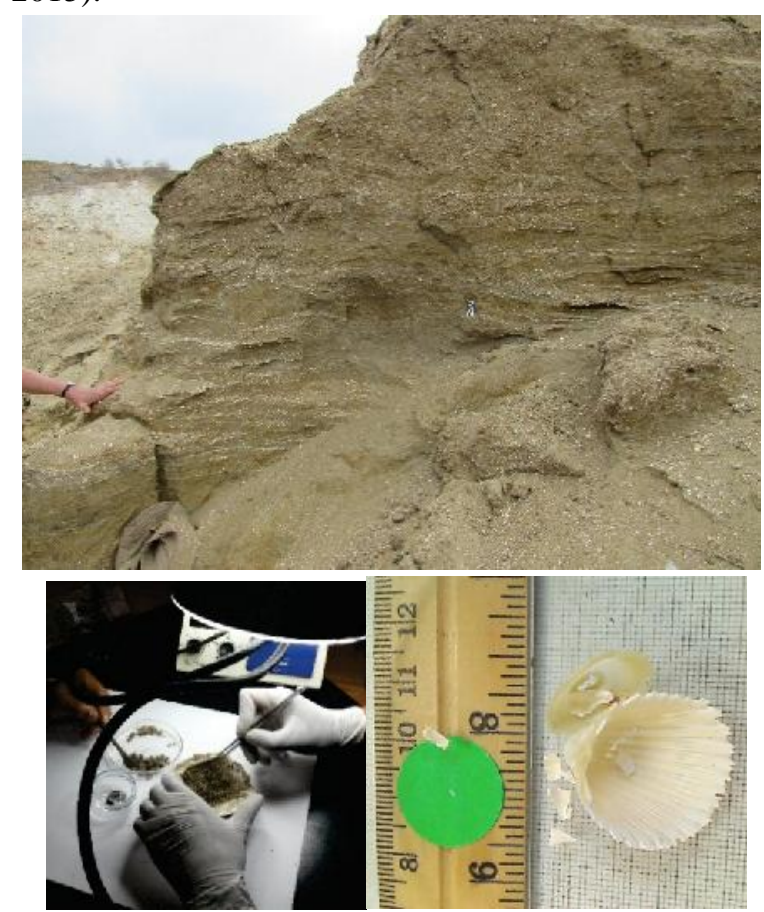

Figura 16. Estación geológica de muestra EJ-02 con moluscos de Trachycardium (Mexicardia) procerum, y el de menor dimensión el Divalinga perparvula, ambas especies de ambiente marino nerítico (Keen, 1971). Datación 14C por el laboratorio Beta Analytic, de $43.245 \pm 460$ años BP.

Los datos de isotopo estable de oxigeno $\delta 180$ referida a la unidad litológica de la estación EJ-02, indica valores de -1 y -1.5 (Figura 17), ambos valores corresponden a dos tipos de muestras ubicadas en 
diferentes distancias pero perteneciente a un mismo nivel sedimentario en la estación EJ-02. La correlación de este dato puede ser interpretado como una etapa interestadial (años cálidos que pueden durar menos de 10.000 años al interior de una época glacial) dentro del periodo glacial del MIS 3 (ver Figura 17), probablemente un rápido ascenso de mar proporcionó aportes sedimentarios marinos y moluscos hace 43.245 años \pm 460 B.P.

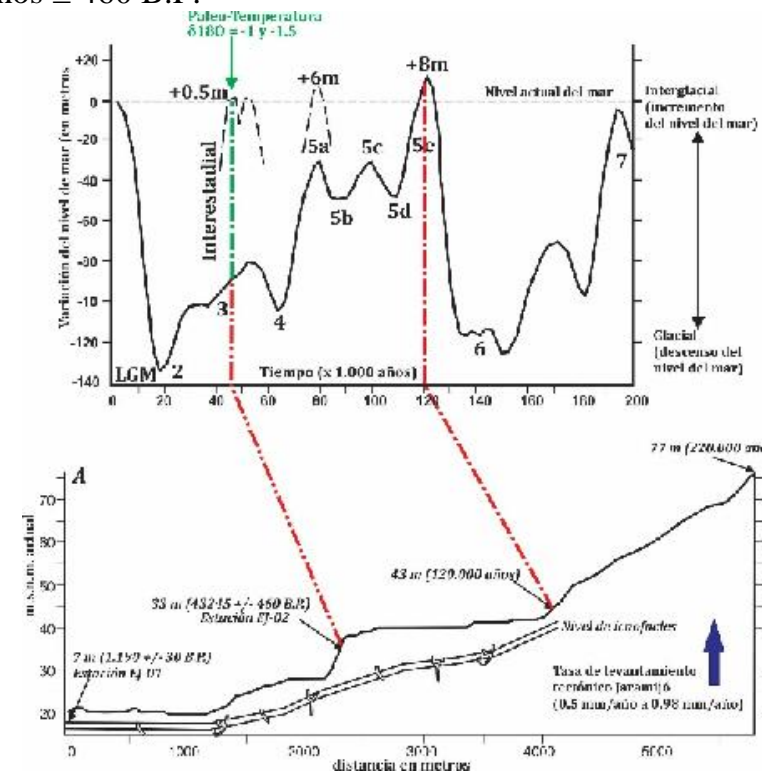

Figura 17. Esquema de terrazas marinas para sitio Jaramijó y confrontación con los MIS 1 a 3. Arriba: Variación de nivel de mar en los últimos 200.000 años (Shackleton, 1978; Martinson et al., 1987) y etapa interestadial en el MIS 3 identificada en este estudio. Abajo: perfil topográfico de terrazas levantadas por tectónica activa $(0.5$ a $0.98 \mathrm{~mm} / \mathrm{año})$. Datación 14C de estación EJ01 desde Mulas et al., 2015.

Las especies de bivalvos (Figura 16) identificadas en este estudio ( 27 en total y descrito su ambiente sedimentario) indican que el nivel EJ-02 durante su fase de sedimentación se encontraba en columna de agua entre los 0 a -30 metros, zona sublitoral de la plataforma interna, esta deducción es confirmada ya que durante el trabajo de campo se buscó estas mismas especies de moluscos en la playa actual, no encontrando los caparazones de bivalvos completos pero sí se encuentran algunos de ellos a una profundidad de columna de agua entre -8 a -10 metros, los cuales fueron observados en el trabajo de mar en lancha donde se extrajo muestras de sedimentos de fondo con el instrumento van veen (Figura 8).

\section{Conclusión}

Esta información ha permitido reconstruir la evolución paleogeográfica y paleoclimática del sitio Jaramijó indicando que los sedimentos cuaternarios y volcánicos presente en el área corresponden a deposiciones de estadios MIS 1 a MIS3, incluyendo el periodo actual interglacial y dos periodos glaciales, y una corta etapa interestadial registrada hace 43.245 años \pm 460 B.P.

La transgresión holocénica y la tasa de retroceso del acantilado estimado es de 11 a 24 metros cada 10 años o dicho en otro sentido de 1.1 a 2.4 metros por año, esto indica la rápida erosión del acantilado conformado litológicamente por sedimentos blandos y roca arcillolita de fácil ripabilidad.

Desde un punto de vista del análisis de la batimetría de la plataforma interna de la zona sublitoral, se puede interpretar como: (a) dos terrazas litorales que son asociadas a paleo-líneas de costa, la distancia entre una de la otra es consecuencia del último avance de la transgresión holocénica en el MIS-1 periodo interglacial actual, pero algo significativo es evidenciado en la rápida tasa asociada desde la paleo línea de costa en el veril $-5.5 \mathrm{~m}$.

\section{Referencias}

[1] Rohling E.J., Grant K., Hemleben CH., Siddall M., Hoogakker B., Bolshaw M., Kucera M. (2008). High rates of sea-level rise during the last interglacial period. Nature geoscience, Vol. 1.

[2] Revell D., Battalio R., Spear B., Ruggiero P., Vandever J. (2011). A methodology for predicting future coastal hazards due to sea-level rise on the California Coast. Climatic Change. Vol 109, Supplement 1, pp 251-276.

[3] Emiliani, C. (1958). Palaeotemperature analysis of core 280 and Pleistocene correlations. Journal of Geology, 66: 264-275.

[4] Shackleton N. (1967). Oxygen isotope analyses and Pleistocene temperatures re-assessed. Nature, 215: 15-17.

[5] Chappell J., Shackleton N. (1986).Oxygen isotopes and sea level. Nature, 324: 137-140.

[6] Martinson D., Nicklas A., Pisias G., Hays J.D. Imbrie J., Theodore C., Moore J. Shackleton N. (1987). Age dating and the orbital theory of the ice ages: Development of a high-resolution 0 to 300,000-year chronostratigraphy. Volume 27, Issue 1, January 1987, Pages 1-29.

[7] Cobb K., Charles D., Cheng H.,Lawrence R. (2003). El Niño/Southern Oscillation and tropical Pacific climate during the last millennium. Nature 424, 271-276. doi:10.1038/nature01779

[8] Levitus S., Antonov J.I., Boyer T.P., Locarnini H, Garcia E., Mishonov A.V. (2009). Global ocean heat content 1955 - 2008 in light of recently revealed instrumentation problems. Geophys. Res. Lett., 36, L07608, doi: 10.1029/2008GL037155.

[9] Mato F., Toulkeridis T. (2017). The missing link in El Niño's phenomenon generation. Science of Tsunami Hazards 36(3):128-144 
[10] deMenocal, P.B. (2001). Cultural responses to climate change during the late Holocene, Science, 292, 667-673, 2001.

[11] Mulas M., Chunga K., Peña E., Falquez D., Lopez Ma., Alcivar R. (2015). Stratigraphic And Lithofacies Study Of Distal Rain-Triggered Lahars: The Case Of West Coast Of Ecuador. AGU Fall Meeting - American Geophysical Union. Session Title: V33B. Eruptive Processes and Watery Hazards of "Wet" Volcanoes on Land, in the Sea, or under Ice III Posters. San Francisco USA. 14/18 December.

[12] Chunga K., Mulas M., Garces D., Quiñónez M.F. (2015). Paleoseismic and Paleogeographic Reconstruction of the Central Coastal of Ecuador: Insight from Quaternary Geological Data for the Jaramijó Bay area. AGU Fall Meeting - American Geophysical Union. Session Title: EP23B. Coastal Geomorphology and Morphodynamics III Posters. San Francisco USA. 14/18 December.

[13] Zeidler, J., Pearsall, D. (1994). Archaeological testing in the lower jama Valley. In: (Eds.), Regional Archaeology in Northern Manabí, Ecuador, Volume 1: Environment, Cultural Chronology, and Prehistoric Subsistence in the Jama river ValleyUniversity of Pittsburgh Memoirs in Latin American Archaeology No. 8, pp. 99e109 (Bilingual volume co-published with Ediciones Libri Mundi, Quito).

[14] Usselman P. (2006). Dinámica geomorfológica y medio ambiente en los sitios arqueológicos Chitije y San Jacinto / Japoto (costa del Manabí central, Ecuador). Bulletin de L'institut Francais d'Etudes Andines, 35 (3): 257-264.

[15] Bristow C.R., Hoffstetter R. (1977). Lexique Stratigraphique, Amérique Latine. Ed. CNRS, Paris, Fas. 5-2, Equateur: 410.

[16] Chunga K., Michetti AM., Mulas M., Besenzon D., Ferrario MF., Garces D., Ochoa F. (2017). Intensidad MAcrosísmica ESI-07 y Efectos Geológicos del Terremoto de Pedernales del 16.04.2016 (Mw 7.8). VIII Jornadas en Ciencias de la Tierra. Quito. Abstract.

[17] Posamentier, H. W., Allen, H. W., James, D. P., and Tesson, M. (1992). Forced regressions in a sequence stratigraphic framework: Concepts, examples, and sequence stratigraphic significance: American Association of Petroleum Geologists Bulletin, v. 76, p. 1687-1709.
[18] Soledispa B. (2012). Caracterización geomorfológica y sedimentológica de la bahía de Jaramijó, en la provincia de Manabí. Acta Oceanográfica del Pacífico. vol. 17, $\mathrm{n}^{\circ}$ 1, 2012. Pag-208.

[19] Pedoja, K., Dumont, J.F., Lamothe, M., Ortlieb, L., Collot, J.-Y., Ghaleb, B., Auclair, M., Alvarez, V., y Labrousse, B. (2006). PlioQuaternary uplift of the Manta Peninsula and La Plata Island and the subduction of the Carnegie Ridge, central coast of Ecuador, Journal of South American Earth Science, 22, 1-21.

[20] Burbank, D.; Anderson, R. 2001. Tectonic Geomorphology. Blackwell Science; 273 p. Estados Unidos.

[21] Fairbridge, R. W. (1976). Shellfish-eating preceramic Indians in coastal Brazil. Science 191, 353-359.

[22] Shepard, F. P. (1963). 35,000 years of sea level. In "Essays in Marine Geology," pp. 1- 10. Univ. Southern California Press, Los Angeles, Calif.

[23] Shackleton N. (1978). Oxygen isotope stratigraphy of the Middle Pleistocene. pp 1-16, In: Shotton, F.W., British Quaternary Studies. Recent advances. Clarendon Press, Oxford.

[24] Quiñonez-Macias M. (2016). Indicadores Geomorfológicos y Bioestratigráficos para la Reconstrucción Paleoclimática de Jaramijó: Potenciales Amenazas Geológicas Registradas en Sedimentos. Tesis de Master. Espol.

[25] Chunga K., Mulas M., Quiñonez-Macias M., Garcés D., Constantine A. (2016). Geomorphologic and Stratigraphic relationships as indicators of Geologic Hazard and Paleoseismicity, central coast of Ecuador. 7th International INQUA Meeting on Paleoseismology, Active Tectonics and Archeoseismology (PATA).

[26] Keen, A. M. 1971. Sea Shells of Tropical West America. Marine mollusks from Baja California to Peru, ed. 2. Stanford University Press. xv, 1064 pp., 22 pls. 